\title{
Validation of food-diary and duplicate plate methods for the estimation of fluoride intake in children
}

\author{
N. Omid ${ }^{1}$, F. V. Zohoori ${ }^{1}$, A. Batterham ${ }^{1}$, W. T. O’Hare ${ }^{1}$ and A. Maguire ${ }^{2}$ \\ ${ }^{1}$ Health and Social Care Institute, Teesside University, Middlesbrough and ${ }^{2}$ Centre for Oral Health Research, \\ School of Dental Sciences, Newcastle University, UK
}

The 3-day food diary (3-d FD) and 2-day duplicate plate (2-d DP) dietary assessment methods have been extensively used for estimating fluoride $(\mathrm{F})$ intake in children and adults. However, neither of these two methods have been validated and evaluated for their accuracy. Since accurate estimation of dietary intake in children is difficult and because dietary assessment methods are associated with measurement errors, urinary nitrogen and potassium have been suggested as biomarkers to provide unbiased measurements of dietary intake in both children and adults ${ }^{(1)}$.

This study aimed to examine the validity of 3-d FD and 2-d DP for the estimation of dietary F intake in children using i) the ratio of urine nitrogen to dietary nitrogen (UN/DN) and ii) the ratio of urine potassium to dietary potassium (UK/DK).

Sixty-one healthy children aged 4-6 year old were recruited. Dietary data were collected using 3-d FD and 2-d DP methods. Twenty-four hour urine samples were collected from each subject twice. Completeness of 24-h urine samples was verified using creatinine excretion $^{(2)}$ and urine flow rate ${ }^{(3)}$. The UN/DN and UK/DK were calculated based on data from intake and excretion of these nutrients. A cut-off point of $<0.2$ for both UN/DN and UK/DK ratios was used to indicate over-reporting of dietary intake while $>1$ was used to indicate under-reporting.

All 24-h urine samples were verified as complete. Dietary data of 58 (95\%) children, when collected by the 3-d FD, were considered valid, while for the 2-d DP method data for $46(75 \%)$ children were viewed as valid. The results indicated that 2-d DP method was prone to under-reporting dietary data. On the other hand, a small percentage $(5 \%)$ of children were found to be over-reporters when dietary data collected by the 3-d FD method.

\begin{tabular}{|c|c|c|c|c|c|c|}
\hline \multirow[b]{2}{*}{ Method } & \multicolumn{2}{|c|}{$\begin{array}{l}\text { Number (\%) of children } \\
\text { with valid data }\end{array}$} & \multicolumn{2}{|c|}{$\begin{array}{l}\text { Number (\%) of } \\
\text { under-reporters }\end{array}$} & \multicolumn{2}{|c|}{$\begin{array}{c}\text { Number (\%) of } \\
\text { over-reporters }\end{array}$} \\
\hline & Number & $(\%)$ & Number & $(\%)$ & Number & $(\%)$ \\
\hline $3-\mathrm{d} F D$ & 58 & (95) & $\overline{0}$ & $(0)$ & 3 & (5) \\
\hline 2-d DP & 46 & (75) & 15 & (25) & 0 & (0) \\
\hline
\end{tabular}

Mis-reporting of dietary intake is a major problem in dietary surveys. The use of urinary and dietary nitrogen and potassium might improve independent validation of dietary intake if included in the protocol of dietary intake studies used in both adults and children. However, the optimal cut-off points for children should be investigated further.

Supported by grants from University of Teesside Research Doctoral Scholarships and The Borrow Foundation.

1. Day NE, McKeown NM, Wong M, et al. (2001) Int J Epidemiol 30, 309-317.

2. Tietz NW (1995) In Clinical guide to laboratory tests, 3rd ed., pp. 188, Philadelphia: WB Saunders Co.

3. Marthaler TM (1999) Monitoring of renal fluoride excretion in community preventive programme on oral health. Geneva: World Health Organisation. 